\title{
Kultur in fachdidaktischen Lehrbüchern - eine diskursanalytische Perspektive auf Kultur in der Lehrerbildung
}

\author{
Claudia Kühn (D) Konstantin Lindner • Annette Scheunpflug
}

Eingegangen: 6. März 2020 / Überarbeitet: 15. Juli 2020 / Angenommen: 28. Oktober 2020 / Online publiziert: 23. November 2020

(C) Der/die Autor(en) 2020

Zusammenfassung Lehrkräfte tradieren Kultur. Mit der dargestellten empirischen Studie wird die Frage, wie „Kultur“ in fachdidaktischen Lehrbüchern der universitären Lehrkräftebildung präsentiert wird, bearbeitet. Lehrbücher der Englisch-, Geschichts- und Musikdidaktik wurden über ein mehrschrittiges Verfahren identifiziert und hinsichtlich kulturbezogener Vorstellungen diskursanalytisch ausgewertet. Die Befunde zeigen, dass der Kontingenz des Kulturellen im Rekurs auf bedeutungsorientierte kulturtheoretische Konzepte begegnet wird, Differenz setzende Aspekte in Bezug auf Praxisvorschläge implizit präsent sind und nur wenige metatheoretische sowie die Lehrkräfte zur Selbstreflexion einladende kulturbezogene Reflexionsangebote gemacht werden.

Schlüsselwörter Diskursanalyse · Kultur · Kulturelle Bildung · Lehrerbildung · Fachdidaktik

\footnotetext{
C. Kühn $(\bowtie)$

Professur für Erwachsenenbildung und Weiterbildung, Otto-Friedrich-Universität Bamberg, Markusplatz 3, 96047 Bamberg, Deutschland

E-Mail: claudia.kuehn@uni-bamberg.de

Prof. Dr. K. Lindner

Religionspädagogik und Didaktik des Religionsunterrichts, Otto-Friedrich-Universität Bamberg, An der Universität 2, 96047 Bamberg, Deutschland

E-Mail: konstantin.lindner@uni-bamberg.de

Prof. Dr. A. Scheunpflug

Lehrstuhl für Allgemeine Pädagogik, Otto-Friedrich-Universität Bamberg,

Markusplatz 3, 96047 Bamberg, Deutschland

E-Mail: annette.scheunpflug@uni-bamberg.de
} 


\title{
Culture in textbooks of subject didactics. Discourse analytical perspectives on culture in teacher training
}

\begin{abstract}
Teachers transmit culture. The present study raises the question of how contexts of culture are presented in didactic textbooks used in university teacher training. To this end, textbooks of English, History and Music Didactics were identified in a multi-step procedure and evaluated using discourse analysis with regard to culture-related concepts. The results show that meaning-orientated cultural-theoretical concepts are used to respond to the contingency of culture, that aspects of difference are implicitly present in relation to proposals for practice, and nearly no offers are made to (prospective) teachers to deal meta-theoretically and selfreflectively with concepts of culture and cultural education.
\end{abstract}

Keywords Culture $\cdot$ Cultural education - Discourse analysis - Subject didactics · Teacher training

\section{Kulturreflexiver Unterricht als Herausforderung der gegenwärtigen Lehrerbildung}

In diesem Beitrag werden Forschungsergebnisse zum Verständnis von Kultur und deren Thematisierung in fachdidaktischen Lehrbüchern der universitären Lehrerbildung präsentiert. Ausgangspunkt der Analysen ist, dass in fachdidaktischen Lehrbüchern explizite und implizite Vorstellungen von Kultur und kultureller Bildung Ausdruck finden, die Studierende für eine kulturreflexive Unterrichtsgestaltung mehr oder weniger sensibilisieren können. Kulturreflexiver Unterricht gewinnt gegenwärtig an Bedeutung, weil die Schule als Kultur vermittelnde und Kultur geprägte Bildungsinstanz herausgefordert ist, Kinder und Jugendliche auf die zunehmende Hybridisierung des Kulturellen vorzubereiten, um in einer sich individualisierenden und pluralisierenden Gesellschaft entscheidungs-, gestaltungs- und handlungsfähig zu sein (Scheunpflug et al. 2012; Costa et al. 2018). Vor diesem Hintergrund gewinnt ein Verständnis von Kultur als ein ,selbstgesponnenes Bedeutungsgewebe“ (Geertz 2010; Reckwitz 2011) an Relevanz, das Optionen bietet, sich zur zunehmenden Kontingenz kultureller Bezugssysteme zu verhalten sowie einen machtreflexiven, dekolonisierenden Blick auf Kultur einzunehmen (Bhabha 2000).

Eine zentrale Aufgabe der Lehrerbildung ist, angehende Lehrkräfte bereits in der universitären Phase darauf vorzubereiten, ,Inhalte, Prinzipien, Gesichtspunkte, Rahmen und Standards schulisch-kultureller Vermittlungsarbeit kritisch und produktiv zu denken und zu behandeln“ (Klepacki 2014). Erste Überlegungen zur Bedeutung von Kultur und kultureller Bildung in der Unterrichtsentwicklung (Stute und Wibbing 2014), Schulentwicklung (Fuchs 2019) sowie zur Schulkultur (Klopsch 2019) liefern im kulturellen Bildungsdiskurs wichtige theoretische und praktische Implikationen für ein Verständnis von Schule als kulturgeprägte und kulturvermittelnde Bildungsinstanz. Bisher fehlt es aber an Forschungen, die dezidiert Aufschluss geben, welche kulturbezogenen Vorstellungen angehenden Lehrkräfte in ihrer Ausbildung präsentiert werden. Insbesondere mangelt es bisher an systematischen Über- 
legungen zur Funktion von Lehrbüchern als kulturbezogene und kulturell bildende Bedeutungsträger.

Die im Folgenden vorgestellte qualitative Studie setzt an diesem Desiderat an. Sie ist Teil des interdisziplinären Forschungsprojekts „Kultur in der Lehrerbildung (KulturLebi)“, das - ausgehend von einem bedeutungsorientierten Kulturbegriff verschiedene Zugänge zum Thema in den Blick nimmt (vgl. zu den Vorstellungen von Lehramtsstudierenden Timm und Scheunpflug 2020; zu der Kulturausübung von Lehramtsstudierenden Costa und Drechsel 2020). In diesem Beitrag werden die empirischen Befunde der Teilstudie „Kultur im Diskurs (KuDis)“ vorgestellt, die sich expliziten und impliziten Kulturverständnissen in fachdidaktischen Lehrbüchern geistes- und kulturwissenschaftlicher Unterrichtsfächer widmet. Nach Darstellung des Forschungsstands und Präzisierung der Fragestellung (Abschn. 2) werden das methodische Vorgehen der Studie (Abschn. 3) und diskursanalytische Befunde zu kulturbezogenen Diskursformationen in englisch-, geschichts- und musikdidaktischen Lehrbüchern präsentiert (Abschn. 4). Ausgehend von den empirischen Ergebnissen werden in Orientierung an einer metareflexiven Professionstheorie (Cramer et al. 2019) Aspekte einer Theorie kultureller Lehrerbildung auf Lehrbuchebene angeregt, die auf die Sensibilisierung einer kulturreflexiven Unterrichtsgestaltung abzielen (Abschn. 5). Abschließend werden Anregungen für Forschung und Praxis gegeben (Abschn. 6).

\section{Forschungsstand: Kultur im Diskurs der Lehrerbildung}

Für die Beschreibung des Forschungsstands sind folgende Diskursstränge von Relevanz:

Kulturelle Lehrerbildungsforschung Im Forschungsdiskurs zur kulturellen Bildung werden in schulischer Hinsicht vor allem ,die Sparten Bildende Kunst, Musik, Literatur und Darstellende Kunst“ (Liebau et al. 2013; vgl. Althans und Audehm 2019) untersucht: Andere - ebenfalls kulturbildende - Sparten und damit verknüpfte Unterrichtsfächer wie Sprachen oder Geschichte bleiben demgegenüber unterrepräsentiert. Ein zentrales Desiderat bildet die kulturbezogene Erforschung geistes- und kulturwissenschaftlicher Unterrichtsfächer in diskursanalytischer und disziplinvergleichender Hinsicht (Klepacki 2012; Liebau et al. 2014).

Diskursanalytische Zugänge zu Fachdidaktiken Ein Blick in die bildungswissenschaftliche Forschung zeigt, dass diskursanalytische Zugänge zu einzelnen kulturbezogenen Aspekten fachdidaktikspezifisch gesucht werden (z. B. Kaiser 2018; Viebrock 2008). Disziplinübergreifend geraten kulturbezogene Facetten wie bspw. unterrichtsbezogene Themenkonstitutionen (z. B. Lange und Sinning 2014a, 2014b) in den Blick. Diskursanalysen zu Kulturverständnissen in fachdidaktischen Lehrbüchern der Lehrerbildung fehlen bisher gänzlich.

Kulturbezogene Diskursanalysen von Schulbüchern In qualitativen Studien werden explizite und implizite Kulturverständnisse von Schulbüchern international 
vergleichend untersucht, insb. die des Geschichts- (Christophe 2014) und Fremdsprachenunterrichts (Fäcke 2016). Thematisch werden kulturbezogene Aspekte wie Nationalität (Fuchs 2011, S. 9 ff.), Gender (vgl. Leifert 2012; UNESCO und GEI 2018) oder Rassismus (Grawan 2014) fokussiert. Während kulturbezogene Tradierungen unterrichtsspezifischer Schulbücher strukturschwacher Länder erforscht werden, mangelt es an einer kulturkritischen Auseinandersetzung mit deutschen Schulbüchern (Christophe 2014, S. 7f.) sowie an Studien, die kulturbezogene Diskurse fachübergreifend rekonstruieren und reflektieren.

Forschungsfrage Um diese Desiderate zu adressieren, wird in der vorgestellten Studie folgende Fragestellung qualitativ bearbeitet: Welche kulturbezogenen Diskursformationen prägen fachdidaktische Lehrbücher ausgewählter geistes- und kulturwissenschaftlicher Unterrichtsfächer im deutschsprachigen Raum?

Mit dieser Forschungsfrage wird eine Perspektive auf die universitäre Angebotsstruktur der Lehrerbildung eröffnet, die auf einem bedeutungsorientierten Kulturverständnis basiert. Clifford Geertz' semiotisches Verständnis von Kultur als einem „selbstgesponnene[n] Bedeutungsgewebe“ (Geertz 2010, S. 9), in das der Mensch verstrickt ist, als einem vielgestaltigen Resultat menschlicher Deutungen von Welt, welches ständig im Wandel begriffen ist und den Individuen durch reflexive Bildungs- und Teilhabeangebote zugänglich gemacht werden muss, ist dabei leitend. Bewusst wird damit auf ein Verständnis rekurriert, das es vermag, auch implizite symbolische Ordnungen, Codes und Sinnkontexte menschlichen Handelns als Teil von Kultur zu betrachten, und die differenz- und abgrenzungsbezogenen Ausrichtungen totalitätsorientierter oder normativer Kulturkonzepte zu vermeiden (Klepacki 2012; Reckwitz 2011). Fachdidaktische Lehrbücher repräsentieren ein diskursiv angelegtes Kondensat universitärer Lehre und somit den jeweiligen Kanon, der angehenden Lehrkräften präsentiert wird. Analysen kulturbezogener Diskursformationen geben Aufschluss über kulturtheoretische Referenzen und Logiken, auf die die Lehrbuchbeiträge explizit rekurrieren und bilden überdies - häufig implizit - kulturbezogene Konzeptionen von Unterricht ab.

\section{Methodisches Vorgehen: Rekonstruktion kulturbezogener Diskursformationen in ausgewählten fachdidaktischen Lehrbüchern}

Im Fokus der Studie steht die Rekonstruktion kulturbezogener Diskursformationen in fachdidaktischen Lehrbüchern.

Qualitatives Forschungsparadigma Um mehr über kulturbezogene Diskursformationen in der Lehrerbildung zu erfahren, die sich in expliziten und impliziten Wissensbeständen fachdidaktischer Lehrbücher abbilden, ist die Studie im qualitativen Forschungsparadigma verortet (Flick et al. 2013). Dieser Verortung wird durch theoriegeleitete Forschungsentscheidungen Rechnung getragen.

Auswahl der Fachdidaktiken Die Datengrundlage der Studie bilden fachdidaktische Lehrbücher, die anerkannte Wissensbestände der Fachdisziplinen exemplarisch 
repräsentieren. Die Samplebildung orientiert sich am theoretischen Sampling der Grounded Theory (Böhm 2012; Glaser und Strauss 2010), deren Adaption in der wissenssoziologischen Diskursforschung etabliert ist (vgl. Keller 2011a, S. 269f., 2011b, S. 83 f.). Um den Blick auf ästhetisch ausgerichtete kulturbildende Kontexte zu weiten, werden fachdidaktische Diskurse der Englisch-, Geschichts- und Musikdidaktik untersucht. Mit Englisch wurde ein in kulturbezogener Hinsicht kaum beforschtes Unterrichtsfach ausgewählt, das für den sprachlichen Bereich steht. Angesichts der zunehmenden Alltagsrelevanz des Englischen, durch Medien, Jugendkulturen, Werbung oder Formen der „Verdenglischung“ (Jahn 2008), wächst die Herausforderung, Schülerinnen und Schüler in die komplexen Bedeutungszusammenhänge englischer Sprach- und Literaturformen einzuführen (Beuter 2019). Die Entscheidung für eine Fokussierung kulturbezogener Diskursformationen der Geschichtsdidaktik gründet in der Pluralisierung von Identitäts- und Heimatkonzepten, in deren Folge die Reflexion der eigenen identitätsstiftenden kulturellen Historizität und eine (de-)codierende Auseinandersetzung mit Geschichtskultur zunehmend an Bedeutung gewinnen (Bauer et al. 2018; Franken und Lindner 2019). Mit der Musikdidaktik wird eine Perspektive auf ein Unterrichtsfach eröffnet, das aus der ästhetisch-bildenden Fächergruppe gewählt ist. Mit den zunehmenden Ästhetisierungsprozessen in Alltag und Gesellschaft, der Globalisierung der Musikmärkte und den damit verbundenen Ausdifferenzierungen von Zugängen zu Musiken wächst der Anspruch an den Musikunterricht hinsichtlich der Zugänge zu Musikkulturen.

Auswahl des Korpus Zur Korpusgenerierung wurden erstens aktuelle Literaturlisten mit Lehrbüchern herangezogen, die an bayerischen Universitäten lehrende Fachdidaktikerinnen und Fachdidaktikern zur Verfügung stellen. Der Fokus auf Bayern verspricht eine kanonbildende Bündelung von fachdidaktikspezifischen Lehrbüchern, da aufgrund der zentralen Staatsprüfung in den jeweiligen Fachdidaktiken eine lediglich standortspezifische wissenschaftliche Fundierung für Lehramtsstudierende Nachteile bei der Bearbeitung der nicht nur am eigenen Universitätsstandort korrigierten Klausuren mit sich bringen würde. Zweitens wurden zur Korpusbildung häufig ausgeliehene Exemplare der Universitätsbibliothek, drittens Literaturlisten und Links zu Online-Angeboten auf den Websites von Fachverbänden und Professuren sowie viertens Literaturhinweise in Ankündigungstexten fachdidaktischer Einführungsveranstaltungen in die Datengrundlage einbezogen. Diese Grundgesamtheit wurde dann wiederum entlang folgender Kriterien reduziert: (1) Mehrfachnennung durch die angefragten Lehrenden waren ein positives Auswahlkriterium, (2) Lehrbücher mit Bezug auf die Primarstufe wurden nicht berücksichtigt und (3) die Bücher, die nicht von allen Lehrenden angeführt wurden, wurden einer intersubjektiven Validierung auf Basis einer kulturbezogene Kontexte fokussierenden Auswertung der Inhalts- und Stichwortverzeichnisse durch die Forschergruppe unterzogen. Im Zuge dieses Auswahlprozesses wurde die Grundgesamtheit von 111 Werken auf einen Korpus von insgesamt sechszehn fachdidaktischen Lehrbüchern in der jüngsten Auflage gebildet, die den entsprechenden Diskurs exemplarisch abbilden und 
eine dezidierte Analyse ermöglichen: fünf geschichtsdidaktische ${ }^{1}$ (A; B; C; D; E), sieben englischdidaktische (F; G; H; I; J; K; L) und vier musikdidaktische Lehrbücher (M; N; O; P) (siehe das Verzeichnis am Ende des Beitrags). Das Verfahren führte dazu, dass in den Korpus vereinzelt auch Lehrbücher aufgenommen wurden, die nicht im engeren Sinne fachdidaktische Einführungswerke sind, sondern eher als Handbücher oder Lehrwerke mit Sammelbeiträgen bezeichnet würden. Dies gründet in deren häufiger Nennung durch die angefragten Dozierenden und deren intensiver Nutzung in der universitären Lehre. Die damit verbundene Diversität wurde in der Auswertung reflektiert.

Wissenssoziologisch fundierte Auswertung Mit dem diskuranalytischen Zugriff in Anwendung qualitativer Methoden und hermeneutischer Interpretationsverfahren wurde ein methodisches Vorgehen aufgegriffen, das in den Kultur- und Sozial- und Erziehungswissenschaften breit rezipiert ist (Keller 2011b, S. 59). Ausgangspunkt ist, dass kommunikativ vermitteltes Wissen das Ergebnis sozialer Konstruktionsleistung ist (Luckmann 2006) und sich solche Wissensbestände als diskursive Formationen abbilden, in denen Regelhaftigkeiten von Aussage, Deutungs- und Handlungspraktiken Ausdruck finden (Angermueller et al. 2014; Foucault 1991 [1971]; Keller 2011a, b). Die ausgewählten Lehrbücher wurden digitalisiert und softwaregestützt auf Diskurselemente hin untersucht, in denen explizite und implizite Wissensbestände des Kulturellen zur Geltung gebracht werden (Dumm und Niekler 2016, S. 99). Diese wiederum wurden mittels Elementen der qualitativen Inhaltsanalyse computergestützt (Sattler 2014) und regelgeleitet analysiert sowie kategorisiert (Mayring 2015, S. 69 ff.). Mittels systematisierend-vergleichender Analyse von Diskurselementen wurden zunächst fachdidaktikspezifische Diskursformationen identifiziert (Keller 2011b, S. 275). Die Relationierung fachdidaktikspezifischer Befunde ermöglichte schließlich die Identifizierung fachdidaktikübergreifender kulturbezogener Diskursformationen. Der Auswertungsprozess wurde in zwei kontinuierlich arbeitenden Interpretationsgruppen intersubjektiv validiert.

Bildung eines kulturbezogenen Kategoriensystems Auf Basis der Auseinandersetzung mit kulturtheoretischen Ansätzen (u. a. Bhabha 2000; Geertz 2010; Reckwitz 2011; Welsch 2010) und erziehungswissenschaftlichen Diskursen (Fuchs 2008; Göhlich 2010; Griese 2006; Wulf 2004) sowie mit dem Material wurden induktiv-deduktiv zunächst am Material aus der Geschichtsdidaktik folgende Kategorien gewonnen: Kultur als (1) subjektiv bestimmtes Geflecht von Deutungen, als (2) Aneignungsprozess, (3) als ein von Differenzsetzungen geleitetes Konstrukt, (4) als machtbezogenes Konstrukt und (5) als hybrides Konstrukt (vgl. ausführlich zum Kategoriensystem Franken und Lindner 2019, S. 189). Die Kodierung wurde mit Hilfe von MAXQDA vorgenommen. Diese insgesamt fünf Kategorien wurden im

\footnotetext{
1 Zu Gunsten der Leserlichkeit werden die einzelnen Werke in den folgenden Ausführungen mit Buchstaben abgekürzt. Vor dem Literverzeichnis wird die Datengrundlage des Korpus gesondert aufgeführt. Dass einzelne Lehrbücher (J; M; N) Sammelwerke aus Beiträgen verschiedener Autorinnen und Autoren darstellen und verschiedene Perspektiven des fachdidaktischen Diskurses abbilden, wurde im Auswertungsprozess reflektiert.
} 
Rahmen intersubjektiver Validierungsprozesse verdichtet, abstrahiert und in eine systematisierende Neuordnung gebracht (Böhm 2012, S. 478) und dann für die weitere Analyse kulturbezogener Schlüsselpassagen aller Lehrbücher des Datenkorpus verwendet. Das Kategoriensystem - das sich auch in Bezug auf die Analyse englisch- und musikdidaktischer Lehrbücher als tragfähig erweist - ermöglicht die Rekonstruktion kulturbezogener Diskursformationen einerseits explizit, insofern auf kulturtheoretische Referenzen rekurriert wird, und andererseits implizit in Ausführungen zu Lehrerinnen und Lehrern, Schülerinnen und Schülern sowie Unterrichtsinhalten (vgl. zur Entwicklung des Kategoriensystems die ausführliche Dokumentation in Kühn et al. 2020). Die Entwicklung des Kategoriensystems wurde ebenfalls durch zwei Interpretationsgruppen begleitet.

\section{Empirische Befunde: Kultur in fachdidaktischen Diskursen}

Im Folgenden werden die empirischen Befunde zu kulturbezogenen Diskursformationen in den ausgewählten Lehrbüchern vorgestellt. Dazu wird auf die expliziten kulturwissenschaftlichen und -theoretischen Referenzen der Fachdidaktiken Bezug genommen (Abschn. 4.1), zudem werden die implizit verankerten kulturbezogenen Diskursformationen entlang des gebildeten Kategoriensystems systematisiert (Abschn. 4.2). Die Ergebnisdarstellung mündet in eine Herausstellung des Potenzials, wie Lehramtsstudierende auf eine kulturreflexive Unterrichtsgestaltung hinsichtlich der Gestaltung fachdidaktischer Diskurse und vor dem Hintergrund eines bedeutungsorientierten Kulturbegriffs vorbereitet werden können (Abschn. 4.3). Die Übersicht über die Kriterien, ihre Merkmale und Textstellenbelege finden sich in Tab. 1 .

\subsection{Explizite kulturwissenschaftliche und -theoretische Referenzen}

In allen untersuchten fachdidaktischen Diskursen erhält der Kulturbegriff explizit Bedeutung. Dabei rekurrieren die Autorinnen und Autoren meist auf ein bedeutungsorientiertes Kulturverständnis, das auf die Hybridisierung des Kulturellen Bezug nimmt: Die Analyse der Lehrbücher verdeutlicht, dass die jeweilige Fachdidaktik im Horizont eines kulturwissenschaftlichen Bezugsrahmens und bedeutungsorientierten Kulturbegriffs, wenn auch häufig weniger systematisch, präsentiert wird. Es zeigt sich zudem, dass sich neben fachdidaktikübergreifenden kulturbezogenen Referenzen fachdidaktikspezifische Kulturkonzepte etabliert haben. Während kaum Bezüge zu transkulturellen Perspektiven zur Geltung gebracht werden, sind Bezüge zur Interkulturalität übergreifend präsent.

Die Geschichtsdidaktik verortet sich - wenn auch für die Rezipienten stärker implizit bleibend - im Bezugsrahmen der Kulturwissenschaften und verweist dabei auf ein bedeutungsorientiertes Kulturverständnis (A, S. 122f.; B, S. 32; D). Für den analysierten Diskurs zeigt sich, dass entweder kulturwissenschaftlich fundierte Kultur-Vergewisserungen grundsätzlich prägend sind (D), die Dimension Kultur kaum in ihrer grundierenden Relevanz thematisiert wird (E) oder kulturbezogene Verortungen versatzstückweise zur Geltung kommen (A; B; C). Alle untersuchten 


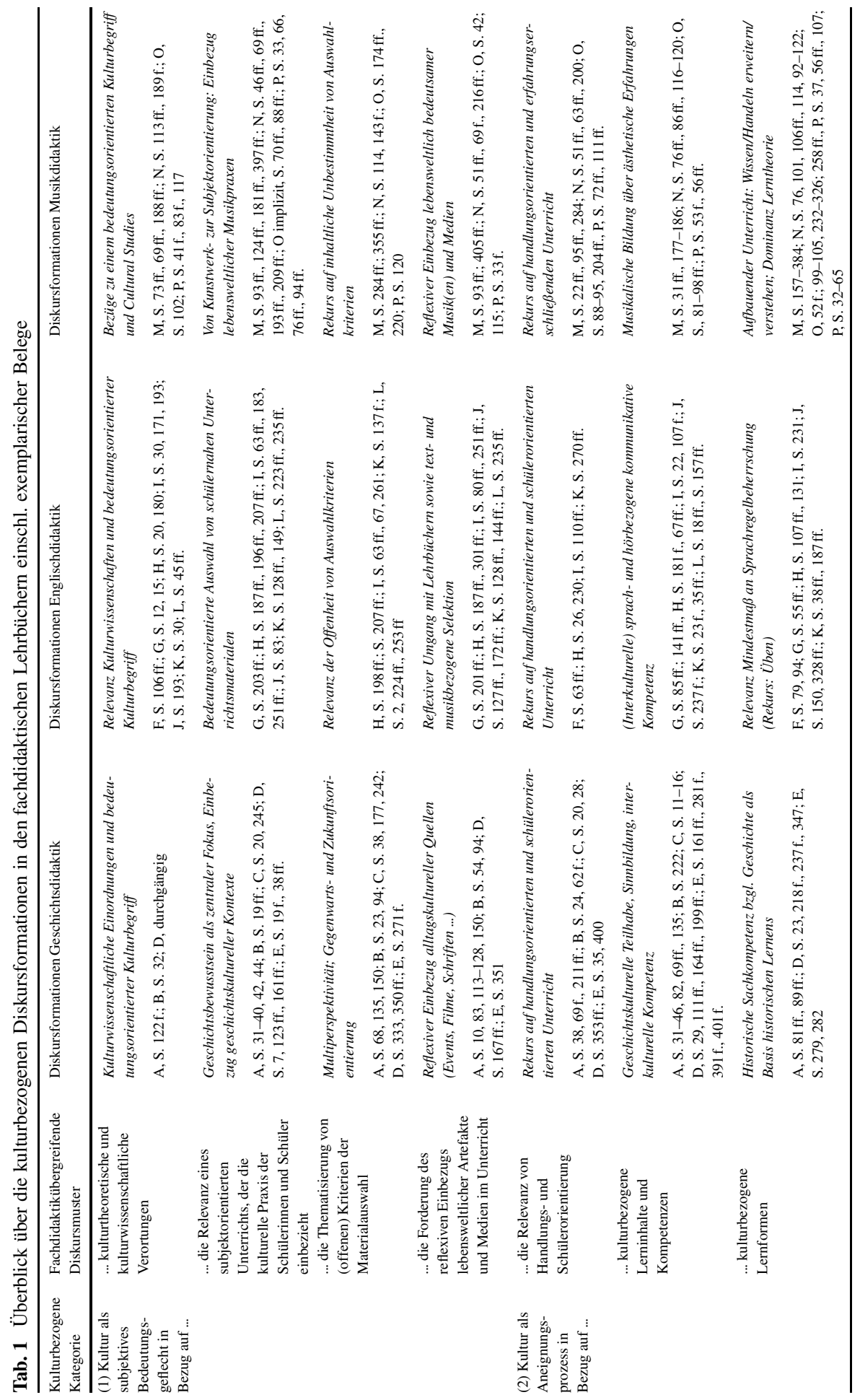




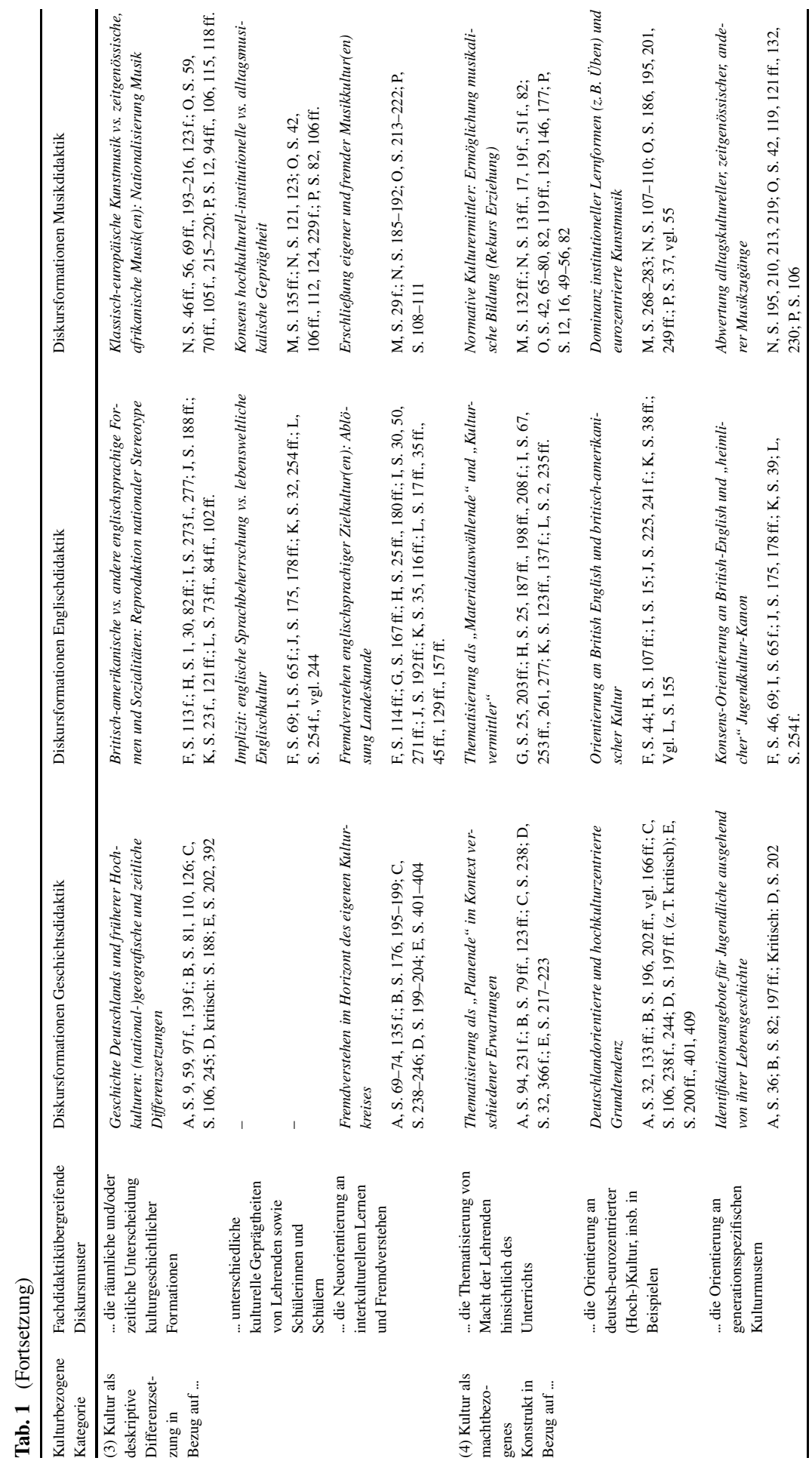




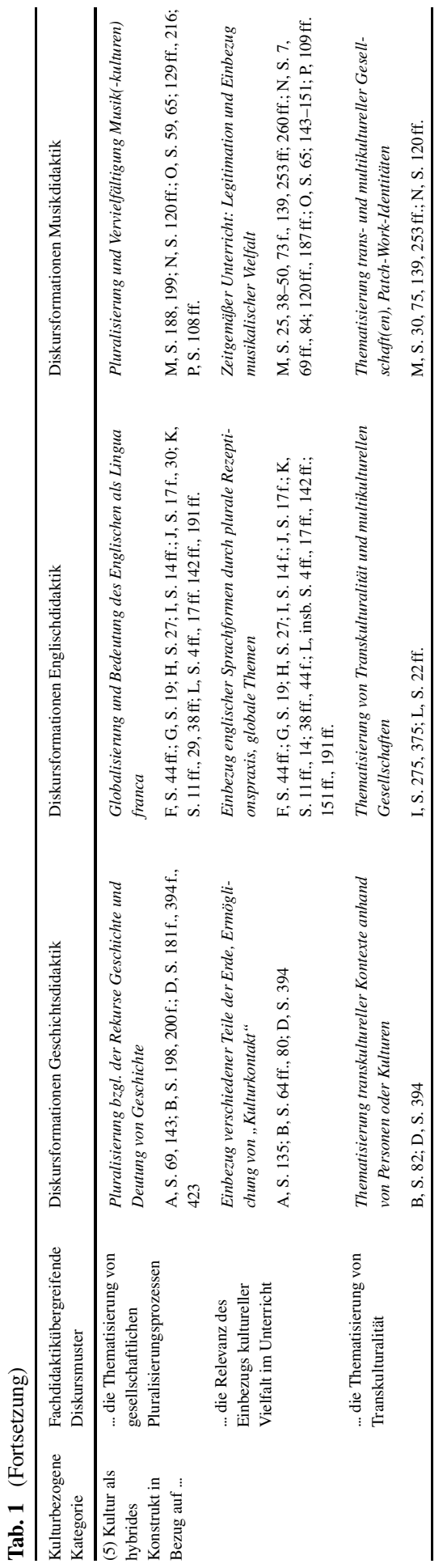


Lehrbücher der Geschichtsdidaktik widmen in der Regel mindestens ein Kapitel dem Fachdidaktik spezifischen Konzept „Geschichtskultur“ und/oder dem „Geschichtsbewusstsein“ (D, S. 111-172; A, S. 31-46; C, S. 11-16; E, S. 391 f.) oder verwenden diese Konzepte selbstverständlich, d.h. ohne weitere Erläuterungen (B, S. 32). In diesem Zusammenhang ist auch der Terminus ,Erinnerungskultur“ präsent (A, S. 43; B, S. 222; D; E, S. $161 \mathrm{ff}$.). Zudem wird verstärkt auf interkulturelles Lernen rekurriert (A, S. 69-74, 135 f.; B, S. 195-199; C, S. 238-246; D, S. 199-204; E, S. 401-404).

In den untersuchten Lehrbüchern wird die Englischdidaktik in unterschiedlichem Umfang und Systematisierungsgrad in einen kulturwissenschaftlichen Bezugsrahmen gestellt. Insgesamt zeigt sich eine Loslösung vom Landeskundekonzept hin zu einer zunehmenden kulturwissenschaftlichen Positionierung des Englischunterrichts: Es wird auf Befunde der Cultural Studies (F, S. 106 ff.; G, S. 15; H, S. 180; I, S. 30, 171; J, S. 193; K, S. 33; L, S. 45 ff.) und ein Verständnis von Kultur als ,,a whole way of life (R. Williams)“ rekurriert, das „Alltagsbereiche und Alltagswirklichkeit, Sprach- und Kulturgewohnheiten“ (G, S. 15) einschließt. Kennzeichnend für den Diskurs ist auch, dass verschiedene Kulturbegriffe sowie damit einhergehende, unterschiedliche Interpretationsweisen präsentiert werden, aber die meisten Lehrbücher für den Umgang mit diesen kaum Orientierung bieten. Zwei Lehrbücher zeichnen sich durch einen reflexiven Umgang mit kulturtheoretischen Referenzrahmen und didaktischen Möglichkeiten aus (K; L; z. T. G). Im übergreifenden Rekurs auf Interkulturalität (F, S. 114 ff.; G, S. 167 ff.; H, 180 ff.; I, S. 271 ff.; J, S. 192 ff.; K, S. $116 \mathrm{ff}$.; L, S. $129 \mathrm{ff}$., $157 \mathrm{ff}$.) und damit verknüpfte Theorien wird ein Kulturkonzept zur Geltung gebracht, das als kulturtheoretische Weiterentwicklung landeskundlicher Wissensvermittlung Relevanz besitzt (indirekt I; J). Insgesamt tritt die Thematisierung (inter-)kultureller Kompetenzen hinter sprach- und hörbezogenen deutlich zurück.

Der musikdidaktische Diskurs zeichnet sich durch eine explizite kulturwissenschaftliche und -theoretische Verortung aus. Alle Werke explizieren den Kulturbegriff, variieren in Umfang und Reflexionsgrad aber erheblich. Bezüge zu einem bedeutungsorientierten Kulturverständnis (M, S. 73 ff., 69 ff.; N, S. 113 ff.; O, S. 102; P, S. 41 f., 117) und zu den Cultural Studies (M, S. 29f, 188 ff.; N, S. 189f.) werden meist hergestellt. Die damit verbundene Relevanz der Ethnomusikologie (P, S. 24) findet im musikdidaktischen Diskurs aber ,explizit erst in jüngster Zeit Erwähnung“ (M, S. 74). Meist werden verschiedene kulturtheoretische Bestimmungsversuche aneinandergereiht und nur wenige Positionierungen sichtbar. Zwei Lehrbücher zeichnen sich durch eine stärkere kulturreflexive Perspektive aus (M; N). Alle Lehrbücher rekurrieren in unterschiedlichem Umfang auf das Interkulturalitätskonzept (M, S. 29 f.; N, S. 185 ff.; O, S. 213 ff.; P, S. 108 ff.), wobei sich ein Lehrbuch explizit gegen eine ethnisch-holistisch geprägte interkulturelle Musikerziehung positioniert (M, S. 30f.). Im Diskurs hat sich zudem der Bezug zur Ermöglichung ästhetischer Erfahrungen etabliert (M; N, S. $113 \mathrm{ff} ., 214 \mathrm{ff} . ;$ O, S. $99 \mathrm{ff}$; P, S. $105 \mathrm{ff}$.). 


\subsection{Systematisierung kulturbezogener Diskursformationen}

Im Folgenden wird das Diskursfeld über die einzelnen Kategorien aufgespannt dargestellt. Die Textstellenbelege sind, der besseren Übersichtlichkeit und Lesbarkeit geschuldet, in Tab. 1 abschließend zusammengefasst dargestellt.

\subsubsection{Kultur als subjektives Bedeutungsgeflecht}

In den Lehrbüchern wird das Verständnis von Kultur als subjektivem Bedeutungsgeflecht aufgenommen, wie es von Geertz (2010) oder Reckwitz (2011) beschrieben wird. Mit dieser Kategorie werden Diskurselemente erfasst, die auf die subjektiven Wahrnehmungs-, Deutungs- und Handlungszusammenhänge des Kulturellen verweisen. Diese Diskursformationen betonen das Deuten und Denken des Einzelnen und nehmen auf die zunehmende Hybridisierung des Kulturellen Bezug. In diesem Kontext werden die kulturellen Prägungen der imaginierten Schülerinnen und Schüler und ein subjektorientierter Unterricht relevant. Es wird ein reflexiver Umgang mit kulturellen Zuschreibungen und eine Auswahl von Unterrichtsmaterialien seitens der Lehrkräfte gefordert, die sich an den Interessen der Schüler und Schülerinnen orientiert. Wie jedoch ein Unterricht angesichts der sich dann daraus ergebenden kulturellen Pluralität aussehen soll, bleibt in der Regel offen. Zwar bieten fachdidaktische Lehrbücher Kriterien für die Auswahl der seitens der Lernenden eingebrachten kulturellen Perspektiven in den Unterricht; inhaltlich und didaktisch offen bleibt jedoch meist, wie und in welchem Umfang schülernahe Unterrichtsinhalte angemessen er- und vermittelt werden können und wie diese in Bezug zu curricularen Perspektiven - für die Geschichtsdidaktik z. B. Multiperspektivität oder Identitätsbildung (B, S. 23; C, S. 38, 177, 242) - gebracht werden.

In der Geschichtsdidaktik geraten subjektive Bedeutungszuschreibungen primär im Geschichtskultur-Konzept in den Blick, das als „soziales System“ (A, S. 42) kommunikativ erzeugt und in multiperspektivisch angelegten Lehr-Lernprozessen hinsichtlich einer „Bildung individuellen Geschichtsbewusstseins geschichtskulturell stimuliert“ (C, S. 20) wird. Dazu wird der reflexive Einbezug alltagskultureller Quellen eingefordert (D, S. 7). In der Englischdidaktik wird ein reflexiver Umgang mit kulturellen Zuschreibungen eingefordert, der einen flexiblen Umgang mit Lehrwerken voraussetzt (G, S. 201 f.; H, S. 301 ff.; I, S. 80 ff.; J, S. 127 ff.; K, S. 144 ff.; L, S. 235 ff.). Dazu wird auf eine Auswahl text- und musikbezogener Unterrichtsmaterialen verwiesen (G, S. 203 ff.; H, S. 187 ff.; I, S. 251 ff.; K, S. 128 ff.; L, S. 235 ff.), die an den Interessen der Schülerinnen und Schülerinnen ansetzt. Es werden aber nur wenige Auswahlkriterien angeboten (H, S. 198 ff., 208f.; I, S. 67, 261; K, S. 137 f.; L, S. 2): Ausgeblendet bleibt meist, wie schülernahe Unterrichtsinhalte angemessen ermittelt und ausgewählt werden können. In der Musikdidaktik beziehen sich die Lehrbücher auf einen subjektiv bedeutungsorientierten Kulturbegriff (M, S. $73 \mathrm{ff}$., 69 ff.; P, S. 41 f., 117; implizit: O, S. 102; N, S. 113 ff.), dieser wird aber nur teilweise umfassend berücksichtigt $(\mathrm{N} ; \mathrm{M})$. Es wird für eine Loslösung von einem kunstwerkorientierten hin zu einem subjektorientierten Musikunterricht plädiert, der den Einbezug lebensweltlich bedeutsamer „musikalischer Gebrauchspraxen“ (N, S. 71) der Schülerinnen und Schüler fordert und dazu auf die inhaltliche Unbestimmtheit 
von Auswahlkriterien setzt (Ausnahme: N). Eine subjektorientierte Unterrichtsgestaltung (M, S. 93 ff.; N, S. 51 ff.; O, S. 42; P, S. 33 ff.) wird jedoch - nach Ansicht der jeweiligen Autorinnen und Autoren - in bisherigen didaktischen ,Modellen und Konzepten nicht befriedigend eingelöst“" (N, S. 65).

\subsubsection{Kultur als Aneignungsprozess}

Von den bedeutungsorientierten Diskurselementen lassen sich jene unterscheiden, die auf die vorgegebene Aneignung kulturbezogener Lerninhalte und -formen abzielen. Im schulischen Kontext spielt gerade die Aneignung von Kultur eine zentrale Rolle (vgl. kritisch Bourdieu 2006; für die Schule ganz allgemein Fend 1980, S. $128 \mathrm{ff}$.) und auch in kulturtheoretischer Perspektive ist die Aneignung des Kulturellen von Bedeutung (vgl. zur ästhetischen Bildung umfangreich thematisiert hinsichtlich Mimesis und Performanz bei Wulf, z. B. 2004, S. 156-190). In vielen Lehrbüchern geht es um die Aneignung von als unveränderbar beschriebenen Formen des Wissens in Form von geschichtlichen Inhalten, englischer Sprache und Literatur oder musikbezogenen Wissensbereichen. Diese Anforderungsstrukturen werden häufig implizit in Aneignungsformen gekleidet, die schüler-, handlungs- und erfahrungsorientierte Unterrichtsformen nahelegen. Damit werden die Schülerinnen und Schüler als handelnde Lernende in den Blick genommen, deren gruppenbezogene Aneignung des Geschichtlichen, Englischen und Musikalischen übergreifend formbezogen kontextualisiert.

Geschichtsdidaktische Verortungen verweisen insb. auf die historische Sachkompetenz als Basis historischen Lernens und die Befähigung zur ,Teilnahme am geschichtskulturellen Diskurs der Gegenwart" (C, S. 20; vgl. auch A, S. 44; B, S. 167; D, S. 164 ff.), der sich Heranwachsenden insb. in Form von Sachbüchern, historischen Filmen und Events erschließt: Hinweise auf die Bedeutung des eigenen, sinnbildenden Erzählens und Schreibens werden im Kontext ,geschichtskulturelle Kompetenz“ (A, S. 38, 82; B, S. 32, 54; C, S. 28; D, S. 23) definiert - u.a. zur Vergewisserung bzgl. der Konstruiertheit von Geschichte. Ein Lehrbuch situiert geschichtskulturelle Kompetenz dagegen vornehmlich auf einer wissensbezogenen, reflexiven Ebene und der damit ermöglichten kompetenten Verhältnisgewinnung zu Geschichte (D, S. 237 f.). Die theoretischen Einordnungen werden nur bedingt in unterrichtpraktische Überlegungen zum historischen Lernen transformiert. In allen Lehrbüchern der Englischdidaktik wird im Kontext handlungs- und schülerorientierter Vermittlungskonzepte hingegen verstärkt auf die Aneignung sprach- und hörbezogener Kommunikationsfähigkeit rekurriert und diese hinsichtlich der Frage eines angemessenen Mindeststandards an Sprachregelbeherrschung entfaltet (H; K). Die Relevanz einer kulturbezogenen Aneignung findet insbesondere mit Blick auf Überlegungen zum Erwerb interkultureller Kompetenz (F; I; L) bzw. zum interkulturellen Lernen (G; H; J; K; L) Ausdruck, die aber in ihrem Umfang hinter jenen der sprach- und hörbezogenen Kommunikationsfähigkeit weit zurückstehen. Sprachästhetische Praxen, wie die eigene Gedichtproduktion werden weniger thematisiert und auch Konkretisierungen, wie ein handlungsorientierter Unterricht - neben medialem Einsatz - realisiert werden kann. Die musikdidaktischen Lehrbücher suchen explizite Bezüge zum Aneignungskonzept zusammenhangslos (O), praxeologisch $(\mathrm{N})$, 
handlungstheoretisch (P) oder subjektorientiert (M). Aneignungsbezogene Referenzen finden sich in Überlegungen zu „Musik als Handlung“ und „Aneignung von Welt“ (M, S. 22, 25; vgl. N, S. 63, 76f. 88, 180, 200, 220; O, S. 52, 204 ff.; P, S. $39 \mathrm{ff}$; $72 \mathrm{ff}, 105)$, zu schülerorientiertem (M, S. 95, 284; N, S. 63 ff.; O, S. $204 \mathrm{ff}$; P, S. $74 \mathrm{ff}$.) und erfahrungserschließendem Musikunterricht (M, S. 95 ff.; N, S. $51 \mathrm{ff}$; O, S. $88 \mathrm{ff}$; P, S. $74 \mathrm{ff}, 111 \mathrm{ff}$.). Erfahrungserschließender Unterricht zielt auf „das Verstehen der Menschen, die mit Musik umgehen“ (N, S. 51) ab. Im Diskurs ist dabei Ästhetik ,als Schlüsselbegriff der Musikpädagogik“ (O, S. 93) relevant. Über ästhetische Erfahrungen sollen Schülerinnen und Schüler musikbezogenes Wissens, Handeln und Verstehen - auch hinsichtlich der eigenen musikalischen Praxis - erweitern und reflektieren lernen. Ein ,,aufbauender Musikunterricht“ (N, S. 92 ff.) wird insgesamt kognitionsbezogen konzipiert und bspw. mit Üben und der übenden Aneignung von musikalischer Objektivationen in Verbindung gebracht.

\subsubsection{Kultur als deskriptive Differenzsetzung}

Mit der dritten Kategorie werden kulturbezogene Diskurselemente fokussiert, die deskriptiv geleitete Differenzsetzungen forcieren und damit Implikationen von Kulturen als in sich geschlossene Gebilde aufweisen. Prominent formuliert wurde dieses in Herders „Kugelmodell“ der Kultur, das von abgeschlossenen kulturellen Entitäten ausgeht (vgl. Herder 2010 [1772]). Reckwitz (2000) spaltet diesen Kulturbegriff nochmals in einen totalitätsorientierten Kulturbegriff, der im Sinne Herders alle kulturellen Äußerungen in ein Konstrukt zusammenfasst, und einen differenztheoretischen Kulturbegriff, der Kultur in einem unterscheidbaren Subsystem verortet. In vielen Lehrbüchern wird - trotz Rekurs auf ein bedeutungsorientiertes Kulturverständnis - Kultur als abgeschlossene Ontologie nationalstaatlichen Ursprungs beschrieben, ohne dass diese Diskrepanz metareflexiv eingeholt würde. Differenzsetzungen kumulieren in der räumlich und/oder zeitlichen Unterscheidung von kulturgeschichtlichen Formationen, der imaginierten kulturellen Geprägtheit der Lehrenden sowie der Schülerinnen und Schüler und der Orientierung an Interkulturalität.

In allen Lehrbüchern der Geschichtsdidaktik wird die Herausforderung „Einwanderungsland“ im Kontext interkulturellen Lernens adressiert: Fokussiert werden Fremdverstehen und Alteritätserfahrung (A, S. 70; B, S. 176; kritisch D, S. 200) sowie die Reflexion des eigenen Blicks auf das Fremde im Horizont eines ,eigenen Kulturkreises“ (C, S. 239ff.). Lösungsvorschläge verlagern die Herausforderung, indem die ,Ausbildung und Einstellung von Lehrkräften mit Migrationshintergrund“ (C, S. 246) oder ein bilingualer, auch auf ,zielsprachliche Kultur“ (A, S. 136) ausgerichteter Unterricht gefordert wird. Trotz der Problematisierung nationalstaatlicher Verengung und einer ,Kulturalisierungsfalle“ (C, S. 240) zeigt sich jene aber implizit, wenn auf unterrichtsbezogener Beispielebene primär Deutschlandorientierte Geschichte illustrierend eingebracht oder eine ,britische Herkunft“ (C, S. 98) von Sachbüchern als beachtenswert markiert wird. Auffällig ist, dass die geschichtsbezogene Differenzsetzung von Kulturen vornehmlich in drei Perspektiven expliziert wird: (National-)Geografisch und zeitlich, ,wenn es sich um ,fremde Kulturen" wie die chinesische oder eine afrikanische oder um zeitlich weit ent- 
fernte Kulturen wie bei der ägyptischen, griechischen und mittelalterlichen“ (A, S. 32; B, S. 196; C, S. 106) handelt, oder in personen- bzw. genderbezogener Hinsicht, wenn es um die Thematisierung von mächtigen oder, einfachen' Personen (A, S. $123 \mathrm{ff} .:$ „Geschichte von unten“ mittels Zeitzeugenbefragungen), Frauen oder Männern etc. geht (A, S. 72). In der Englischdidaktik sind nationale Differenzsetzungen hinsichtlich britischer und weiterer englischer Sprachformen und -inhalte präsent. Obwohl nationalstaatliche Formationen selten explizit diskutiert werden (L, S. $73 \mathrm{ff}$.), sind britisch-angloamerikanische Beispiele und landeskundliche Vorstellungen (insb. F) implizit dominant. Mit der Ablösung vom Landekundekonzept hin zum interkulturellen Englischunterricht (K, S. 116) wird auf das (Fremd-)Verstehen englischsprachiger ,Zielkulturen“ (G, S. 167) rekurriert und bspw. angenommen, dass Verständigungskompetenzen , von Kultur zu Kultur unterschiedlich“ (L, S. 157) sind. Englischunterricht soll zur dezentralisierenden Sensibilisierung für die eigene und fremde Kultur sowie zu einem respektvollen Umgang mit sprachlichen und kulturellen Unterschieden befähigen (H, S. 182 ff.; I, S. 272 f.; K, S. 117 ff.; L, S. 165 ff.). Die Vorstellungen ,eigener und fremder Kultur“ (F, S. 115) und ,grundsätzlich weiter existierenden Unterschieden von Kulturen“ (L, S. 43) stehen einer „Öffnung gegenüber der anderen Kultur“ (I, S. 183) damit eher entgegen. Präsent sind zudem Überlegungen zum Umgang mit Stereotypen, wobei didaktisch häufig auf eine Reproduktion abgezielt und auch, ,gegen eine Überbetonung der Überwindung von Stereotypen“ (I, S. 273f.) argumentiert wird. In der Musikdidaktik zeigen sich diese Differenzsetzungen hinsichtlich der zeitbezogenen und räumlichen Unterscheidung von verschiedenen Musik(-kulturen) bzw. „Arten von Musik“ (P, S. 86). Es geraten klassische oder zeitgenössische Musikstile und Jugend(-musik-)kultur (-en) (M; N, S. 69 ff.; implizit: P, S. 106, 115; O) sowie europäische Kunstmusik oder Jazz-, Rock- und Popmusik (M; N, S. $209 \mathrm{ff}$.; O) und afrikanisch-körperbasierte Musiken $(\mathrm{N})$ in den Blick. Beispiele beziehen sich primär auf europäische Kunstmusik. Sie werden zudem durch die Orientierung an einem interkulturellen Unterricht befördert, der Vorstellungen eigener und fremder Musikkultur(en) sowie nationalstaatliche Bezüge impliziert: Ziel ist, die ,Vielfalt und Unterschiede der Musikkulturen der Welt vergleichend herauszustellen, aber auch deren Berührungspunkte“ (O, S. 218). „Grenzüberschreitungen“ werden thematisiert (insb. M, S. 208; vgl. O), aber kaum Umgangsweisen für den Unterricht vorgestellt (N, S. 71, 121). Angenommen wird zudem, dass eine ,große Differenz zwischen den musikalischen Biographien von Musikstudierenden und [dem] zukünftigen Klientel“" (M, S. 135) besteht (O, S. 124, 230; P, S. 106f.). Dass die europäische Kunstmusik als das Eigene der Lehrkräfte den lebensweltlichen Musikzugängen der Schülerinnen und Schülern gegenübersteht, wird thematisiert (vgl. M, S. 139f.).

\subsubsection{Kultur als Differenzsetzung, die Machtstrukturen impliziert}

Die beschriebenen essentialisierenden Differenzsetzungen implizieren häufig Machtstrukturen. Der kulturwissenschaftliche Diskurs der letzten Jahrzehnte hat diese impliziten Machtstrukturen des Kulturellen mit ihren Über- und Unterordnungsstrukturen deutlich herausgearbeitet (vgl. Bhabha 2000; Bourdieu 2006; Spivak 1988). 
Machtverhältnisse seitens der Lehrenden werden teilweise expliziert, sie finden jedoch häufig implizit in Ausführungen Ausdruck, die sich auf die konkrete Unterrichtsgestaltung beziehen: Die implizite Orientierung an deutsch-eurozentrierter (Hoch-)Kultur und generationsspezifischen Kulturmustern steht einer offenen Unterrichtsgestaltung entgegen.

In der Geschichtsdidaktik zeigt sich eine deutschlandorientierte Tendenz, wenn die nationalstaatliche Geschichte u. a. mit der „kulturellen Ordnung“ (A, S. 59) einer Gesellschaft legitimiert wird, um Reflexivität der Menschen im Umgang mit Geschichte - z. B. „Nationalfeiertag“ (A, S. 9) - anzubahnen oder „,die gegenwärtige Geschichtskultur in unserem Land mitzugestalten“ (E, S. 392). Dieser Referenzraum soll auch den Schülerinnen und Schülern mit Migrationshintergrund als Identifikationsoption geöffnet werden, wobei diese primär an deutschlandorientierten Kontexten zu biografischen Lernprozessen kommen sollen, z. B. im Horizont der Auseinandersetzung mit dem Holocaust (B, S. 197 ff.; kritisch: D, S. 202). In einem Lehrbuch wird überdies antizipiert, dass „Interkulturelles Lernen [...] in der deutschen Gesellschaft nur bedingt konsensfähig" (C, S. 245) sei. Auffällig ist zudem die Orientierung an „Hochkulturen“ bzw. ,kulturellen Errungenschaften“(B, S. 81; kritisch: D, S. 188). Es finden sich „Macht“ problematisierende Entfaltungen zur ,enthumanisierten Ideologisierung der Geschichte“ (D, S. 32) und Forderungen, ,dass Menschen verschiedener kultureller ,Zugehörigkeit" gleichberechtigt mit- und voneinander etwas über ihre kulturellen Bezugssysteme und Bedingtheiten lernen“ $(\mathrm{C}$, S. 238) sollen. In den Lehrbüchern der Englischdidaktik wird hingegen plädiert, die „Grammatik an den Normen des britischen und amerikanischen Englisch“ (K, S. 14) auszurichten, wobei sie selbst dem British English verhaftet bleiben und die Verdrängung weiterer Sprachformen des Englischen kaum reflektiert wird. Eher wird lernzeitpragmatisch argumentiert, sie nicht sprachbezogen, sondern inhaltlich aufzulösen (I, S. 15; K, S. 14; L, S. 191 ff.). Neben dem Vorschlag, globale Themen einzubeziehen, bleiben Hinweise zu einer reflexiven Auswahl von Jugendliteratur und -musik inhaltlich unbestimmt (H, S. 196 ff., 207 ff.; I, S. 63 ff.; K, S. 149). Trotz der Forderung einer reflexiven Auswahl englischsprachiger Jugendliteratur und -musik bleibt in Beispielen ein angloamerikanischer Kanon präsent und Jugendkulturen werden stärker ausgeblendet, was auf generationsspezifische Deutungsmuster verweist (vgl. L, S. 244f.). In den Werken der Musikdidaktik sind Machtstrukturen dann erkennbar, wenn bestimmte Musik-Räume implizit abgewertet werden, indem bspw. die Frage aufkommt, ob ,deutsche Kinder überhaupt türkische, griechische u. a. Lieder singen“ (O, S. 221) wollen. Machtmomente zeigen sich auch, wenn vermeintliche Alltagserfahrungen der Schülerinnen und Schüler dem systematischen Lernen im Unterricht gegenübergestellt werden: ,möglichst ohne Theorie, ohne Aneignung von Wissen, ohne mühsamen Erwerb von Fertigkeiten und historische Vergewisserung soll Musik ,Spaß“ bringen“ (O, S. 132). Die implizite Abwertung der musikalischen Alltagskultur der Schülerinnen und Schüler (N, S. 219; O, S. 121 ff.) deutet sich auch in der Relativierung einer inhaltlichen Offenheit des Unterrichts an (O, S. 174 ff.; P, S. 28 ff., 102f.): „Zu welcher Musik soll geführt werden? Zur Kunstmusik? Zur Musik fremder Kulturen? Zur Musik der Gegenwart?“ (P, S. 12; vgl. O, S. 20, 174; P, S. 12, 121 ff.). 


\subsubsection{Kultur als hybrides Konstrukt}

Mit der fünften Kategorie geraten letztlich kulturbezogene Diskurselemente in den Blick, die Bezüge zu Pluralisierung, Uneindeutigkeit und Mehrfachzugehörigkeit (vgl. Reckwitz 2008, 2017) herstellen und an transkulturelle Perspektiven (Welsch 2010) anknüpfen. In den Lehrbüchern finden sie hinsichtlich der Thematisierung gesellschaftlicher Pluralisierungsprozesse und transkultureller Perspektiven Ausdruck, werden aber nur selten in Bezug zur konkreten Unterrichtsgestaltung gestellt und geraten in Beispielen fast gänzlich aus den Blick. Hybride und transkulturelle Vorstellungen werden in den Lehrbüchern insgesamt größtenteils marginalisiert.

In Geschichtsdidaktik-Lehrbüchern spielen hybride Vorstellungen eher eine marginale Rolle. Insbesondere bezüglich bilingualem Geschichtsunterricht, der u.a. in Referenz zu transnationalen historischen Konzepten zu konzipieren sei, oder hinsichtlich der Idee „Kulturkontakt“ (B, S. 80: Kleopatra, die durch ihre Lebensgeschichte verschiedene Kulturräume verknüpft) werden sie referenziert. Implizit wird auf transkulturelles Lernen verwiesen, wenn bspw. Klassen thematisiert werden, in denen Kinder aus verschiedenen Weltregionen lernen. Eine entsprechende kulturtheoretische Rückbindung und darauf gründende Umsetzungsbeispiele bleiben jedoch aus (B, S. 82). Zudem finden sich Reflexionen zur Relevanz transkultureller Vergleiche, die auf der Praxisbeispielebene jedoch in kultur- und staatsgebildebezogenen Differenzsetzungen gründen (D, S. 394). In der Englischdidaktik finden Bezüge zu hybriden Vorstellungen des Kulturellen in der Thematisierung von Globalisierungsprozessen und der wachsenden Bedeutung des Englischen als ,lingua franca“" Ausdruck, die von einer eher zusammenhanglosen Erwähnung $(\mathrm{G} ; \mathrm{H})$, bis hin zu einzelnen Unterkapiteln (F; I, J) oder einer konsequenteren Reflexion für den Unterricht $(\mathrm{K} ; \mathrm{L})$ reicht. Konsens scheint, dass auf „,sprachliche Vielfalt (...) der Unterricht angesichts der begrenzten Zeit (...) nur sehr oberflächlich vorbereiten“ (K, S. 39) kann und auf eine „plurale Rezeptionspraxis“ (I, S. 15) sowie den Einbezug globaler Themen gesetzt wird. Positionen für und gegen eine glokale Präsentation werden ebenso selten diskutiert (K; I) wie International oder Global English-Sprachformen (K; L), die zum Teil explizit abgewertet werden (F). Transkulturelle Theorieperspektiven (I; L) bleiben größtenteils aus. In Beispielen spiegelt sich die Vielfalt englischsprachiger Sozialitäten, Menschen und Praxen kaum wider und der Einbezug der Eigenarten des Englischsprechens durch Schülerinnen und Schüler wird kaum in Bezug auf eine Einübung globaler (Hör-)Verständigung reflektiert (K, S. 44f.). In der Musikdidaktik wird hingegen auf die Vielfalt von Musik(en) verwiesen. Alle Lehrbücher thematisieren die Bedeutung gesellschaftlicher Pluralisierungsprozesse für einen zeitgemäßen Musikunterricht (M, S. 199, 188; N; O, S. 65; 129 ff.; P, S. 108 ff.), diskutieren sie aber kaum als konkrete Herausforderung $(\mathrm{M}, \mathrm{S}$. 139) umfassender $(\mathrm{N})$. Während sich an Legitimationen des Unterrichts explizit abgearbeitet wird (M; N; O; P), wird eine plurale Präsentation des Musikalischen im Unterricht inhaltlich-methodisch (N, S. 69 ff., $120 \mathrm{ff}$.) oder kulturwissenschaftlich (M, S. 253 ff.) teilweise differenziert diskutiert. Ein Unterrichtskonzept fokussiert die „Vertrautheit im Umgang mit neuer Musik in ihrer Pluralität“ und „Musik der einen Welt“ (N, S. $187 \mathrm{ff}$.). Transkulturelle Theoriebezüge sind im Horizont multikultureller Gesellschaften und „Patchwork-Identitäten“ (M, S. 253; N, 
S. $120 \mathrm{ff}$.) relevant. In den Darstellungen mangelt es aber an Kohärenz, sodass homogene Vorstellungen des Kulturellen immanent bleiben. Insgesamt wird sich vom Transkultur-Diskurs zugleich abgewendet, wenn ,ein bedeutungsorientierter Kulturbegriff die Frage der Vorsilbe (inter-, multi,- trans- oder hyperkulturell) irrelevant macht, da dieser ohnehin eine transkulturelle Bestimmung der Gesellschaft und des Individuums voraussetzt““ (M, S. 30).

\subsection{Zusammenfassung der Befunde}

Empirisch wird deutlich, dass sich in fachdidaktischen Diskursen der untersuchten Lehrbücher die Pluralisierung des Kulturellen überwiegend - wenn auch in unterschiedlicher Gradualität und Reflexivität - niederschlägt (vgl. die zusammenfassende Darstellung in Tab. 1). Es werden Bezüge zu Kulturtheorien gesucht, die auf einen bedeutungsorientierten Kulturbegriff verweisen, aber nicht umfassend bedacht: Implizit bleiben kulturbezogene Rekurse präsent, die auf ein differenzsetzendes und von Machtpositionen durchzogenes essentialisierendes Kulturverständnis hindeuten. Konkrete Vorschläge zum Umgang mit kultureller Heterogenität im Unterricht bleiben marginal.

Im geschichtsdidaktischen Diskurs werden kulturbezogene Zusammenhänge erstens vor allem unter geschichtskulturellen Aspekten berücksichtigt. Die Teilhabe daran wird zweitens im Sinne einer geschichtskulturellen Kompetenz forciert. Drittens zeigt sich die Tendenz, dass die Thematisierung von Geschichte in kulturbezogener Hinsicht verfestigend wirkt, wenn diese nationalgeografisch und zeitlich kulturalisiert wird. Viertens lässt sich eine deutschland- und hochkulturzentrierte Grundtendenz diagnostizieren, wobei eine Problematisierung dieser Engführung eher ausbleibt (Ausnahme: D). Transkulturelle Perspektiven werden fünftens zaghaft angespielt, durch Umsetzungsvorschläge jedoch, die differenzsetzende Vergleichsperspektiven fokussieren, nicht eingeholt. Um angehende Geschichtslehrende in kulturreflexiver Hinsicht zu sensibilisieren, könnten die Relevanz einer glokal ausgerichteten Einbettung der Geschichtskultur vor Ort, eine stärkere Reflexion der künstlichen Trennung eigener und fremder Kulturen oder Fragen der Justierung des Geschichtekanons angesichts der zunehmend pluralen Schülerschaft deutlicher entfaltet werden. Insbesondere in der reflexiven Schulung der Lehramtsstudierenden bzgl. ihres eigenen Geschichte- und Kulturverständnisses zeigen sich LehrbuchDesiderate.

Für den Englischdidaktik-Diskurs lässt sich resümieren, dass erstens subjektive Bedeutungszusammenhänge der Schülerinnen und Schüler im Horizont eines subjektorientierten Unterrichts relevant sind, aber in kulturbezogener Hinsicht meist unbestimmt bleiben. Zweitens kumulieren Aneignungsperspektiven in einer schülerorientierten Vermittlung kulturbezogener Kommunikationsfähigkeit. Drittens wird ein differenzsetzendes Verständnis in der Unterscheidung englischer Sprachformen und -inhalte sowie der antizipierten Kulturbezogenheit von Lehrkräften und Lernenden sichtbar und viertens eine sprachbezogene und inhaltliche Ästimation des Oxford English sowie britisch-angloamerikanischer Bezüge. Fünftens wird zwar ein Verständnis des Englischen als Lingua franca angedacht, aber Transkulturalität in theoretischen Rekursen und unterrichtsbezogenen Ausführungen größtenteils aus- 
geblendet. Um angehende Englischlehrende auf einen kulturreflexiven Unterricht vorzubereiten, müsste eine glokale Präsentation des Englischen als Lingua franca gezielter mitgedacht, zum reflexiven Umgang mit kulturellen Differenzsetzungen angeleitet, eine Loslösung von nationalen Vorstellungen umfassender eingelöst sowie auf die Repräsentanz nicht britisch-amerikanischer Literatur- und Sprachformen, die Problematisierung der ,verdrängenden“ Dominanzstellung und transkulturelle Perspektiven stärker abgezielt werden (Costa et al. 2018). Obwohl sich die kulturreflexive Ausrichtung des Englischunterrichts in den analysierten Lehrbüchern niederschlägt, wird in den unterrichtsbezogenen Ausführungen ein bedeutungsorientiertes Kulturverständnis implizit beschränkt.

Für den Musikdidaktik-Diskurs zeigt sich erstens, dass subjektive Bedeutungszusammenhänge bzw. musikalische Gebrauchspraxen der Schülerinnen und Schüler im Horizont eines meist subjektorientierten Musikunterrichts relevant sind und dabei zweitens aneignungsbezogene Referenzen in Überlegungen eines schüler- und handlungsorientierten sowie erfahrungsbasierten Musikunterrichts kumulieren. Drittens wird auf die Unterscheidung europäischer Kunstmusik und anderer Musikkulturen, das interkulturelle Verstehen eigener und fremder Musikkulturen und auf eine Differenz der musikalischen Geprägtheit von Lehrenden und Lernenden rekurriert. Machtbezogene Referenzen zeigen sich viertens in der impliziten Orientierung an europäischer Kunstmusik und institutionellen Lernformen. Hybride Vorstellungen sind fünftens im Horizont von Pluralisierung relevant, werden aber nicht weiterführend reflektiert. Eine kulturreflexive Gestaltung des Musikunterrichts würde voraussetzen, dass angehende Lehrkräfte in den Lehrbüchern auf musikbezogene Ausdifferenzierungen und glokale Durchdringungen musikalischer Praxen, die Vielfalt musikalischer Inhalte und Formen, alltagskulturelle Bedeutungszusammenhänge im Unterricht und musikbezogene Differenzsetzungen reflexiv vorbereitet werden. In den analysierten Lehrbüchern wird eine zukunftsfähige Neupositionierung des Musikunterrichts fokussiert, aber in den unterrichtsbezogenen Ausführungen zu wenig reflexiv einholt.

\section{Implikationen für eine Theorie kultureller Lehrerbildung auf Lehrbuchebene}

Diese Befunde regen Konturen einer Theorie kultureller Lehrerbildung auf Lehrbuchebene an. Dazu werden im Folgenden theoretische Anregungen in Bezugnahme auf das offene Konzept der Meta-Reflexivität - als konstitutives Merkmal von Professionalität im Lehrberuf (Cramer et al. 2019) - systematisch beschrieben. Metareflexivität verweist dabei auf die „Kenntnis unterschiedlicher, auf den Lehrberuf bezogener theoretischer Zugänge und empirischer Befunde, die Fähigkeit, diese mit Blick auf ihre jeweiligen Grundlagen und Geltungsansprüche verorten, in ein Verhältnis setzen und sich kritisch mit ihnen auseinandersetzen zu können sowie konsistente, exemplarisch-typisierende Deutungen des komplexen Handlungsfeldes Schule vornehmen zu können" (Cramer et al. 2019, S. 410). Mit Blick auf die empirischen Ergebnisse haben sich folgende Aspekte der Theorie der Metareflexivität 
als bedeutsam erwiesen: Metaperspektivität, Metakommunikation; Distanzierung, Transparenz, Dynamik, Begründungspflicht und Kontextgebundenheit.

Ausgehend von dieser theoretischen Grundierung werden für eine Theorie fachdidaktischer kultureller Bildung die metatheoretische Reflexion des Kulturellen (Abschn. 5.1), die kulturtheoretisch fundierte Interdisziplinarität (Abschn. 5.2) wie auch die Verknüpfung von Kulturtheorie, Unterrichtspraxis und Biografie (Abschn. 5.3) kurz abschließend skizziert.

\subsection{Metatheoretische Reflexion des Kulturbezugs}

Empirisch zeigt sich, dass sich alle analysierten fachdidaktischen Einführungswerke explizit auf den Kulturbegriff beziehen. Angehenden Lehrkräften werden kulturbezogene Vorstellungen präsentiert, die auf den „,cultural turn“ der Kulturwissenschaften verweisen und die zunehmende Pluralisierung und Hybridisierung des Kulturellen mitdenken. Deutlich wird aber auch, dass eine metaperspektivische Betrachtung der Kulturbezogenheit - insofern sie als solche erkenntlich wird - in fachdidaktischen Lehrbüchern häufig als additive Aneinanderreihung kulturtheoretischer Referenzen ihren Niederschlag findet. Nur selten wird die Relevanz eines bedeutungsorientierten Kulturverständnisses als Ausgangspunkt einer zukunftsfähigen Unterrichtsgestaltung ersichtlich und in den Ausführungen entsprechend konsequent reflektiert. Eine konsequente mehrperspektivische Betrachtung der behandelten Gegenstände, die verschiedene Modelle für die Unterrichtsgestaltung reflexiv abwägt, bleibt meist aus. Diese fehlenden praxisbezogenen Brückenschläge zu kulturtheoretischen Positionierungen geben damit nur wenig Orientierung für die Integration kulturbezogener Erkenntnisse in die unterrichtliche Praxis. Eine systematische, metatheoretische (Selbst-)Reflexion bzgl. des Kulturellen, die eine nachvollziehbare und begründete Kritik an bestimmten Kulturtheorien voraussetzt, bleibt ein Desiderat.

Lehrbücher sind Erinnerungstexte, die ,unübersehbar die Signatur der Gegenwart tragen, in der sie entstanden sind. Gleichzeitig leben in ihnen zumindest rudimentär immer auch die Deutungsmuster früherer Zeiten fort“ (Christophe 2014, S. 7) und damit auch tradierte Vorstellungen des Kulturellen. Damit wird die „Distanzierung als Erkennen des Mehrwerts kritisch-konstruktiver, d. h. distanzierter" (Cramer et al. 2019, S. 415) Betrachtungen in kulturbezogener Hinsicht eine Herausforderung. Vor diesem Hintergrund müssten explizite Distanzierungen ermöglicht bzw. zu diesen ermutigt werden.

In den meisten untersuchten Lehrbüchern mangelt es an Explikationen, wie mit den Facetten von Kulturtheorie - im Sinne einer Metakommunikation (Cramer et al. 2019, S. 416) - konkret umgegangen werden kann (Ausnahme: D; L; M). Von daher wäre eine intensivere Auseinandersetzung mit der Relevanz des Kulturbegriffs und kulturtheoretischen Bezüge wünschenswert.

\subsection{Kulturwissenschaftlich fundierte Interdisziplinarität}

Empirisch zeigt sich, dass die jeweilige Fachdidaktik in den analysierten Lehrbüchern im Kontext von Kulturwissenschaften verortet wird. Dies bleibt meist lose für 
sich stehen und Aspekte einer disziplinübergreifenden Kulturbezogenenheit werden wenig mitgedacht.

Die reflexive Bearbeitung eines fachdidaktik- und disziplinübergreifenden bedeutungsorientierten Kulturverständnisses im Horizont von Transparenz und Dynamik (Cramer et al. 2019) verspricht Potenzial, um der voranschreitenden Pluralisierung und Hybridisierung des Kulturellen gerecht zu werden. Eine fächerunabhängige Positionierung zu einer kulturwissenschaftlich fundierten Gestaltung fachdidaktischer Lehrbücher wäre bedeutsam, um dazu beizutragen, eine vom Einzelfall losgelöste generische Kompetenz der Lehrerinnen und Lehrer im Umgang mit hybrider Kulturalität anzubahnen. Damit verbunden wäre eine kulturbezogene Ausrichtung eines hochschuldidaktischen Programms, das zu einem kulturreflexiven Austausch in der Lehrerbildung ermutigt, indem bspw. Lehrbücher und Lehrveranstaltungen in kulturbezogener Hinsicht stärker interdisziplinär vernetzt werden. Lehrkräfte studieren in der Regel mehrere Disziplinen. Hier zwischen den Disziplinen Querverbindungen aufscheinen zu lassen, erzeugt eine dichtere Reflexivität, als dies mit vereinzelten Theorieangeboten der Fall ist. Dass sich die Konfrontation mit der Offenheit des Kulturellen als auch deren Konsequenzen für eine zukunftsfähige Unterrichtsgestaltung „als komplexitätssteigernde Zumutung" (Cramer et al. 2019, S. 416) für Lehramtsstudierende erweisen dürfte, liegt auf der Hand. Gerade vor diesem Hintergrund ist eine interdisziplinär gestützte Herangehensweise von Bedeutung.

\subsection{Verknüpfung von Kulturtheorie, Unterrichtspraxis und Biografie}

Empirisch zeigt sich, dass kulturtheoretische Überlegungen nur bedingt vor dem Hintergrund der Unterrichtspraxis und der Biografie der Akteure konzipiert werden. Insgesamt geraten die Studierenden - als primäre Adressaten der fachdidaktischen Lehrbücher - aus dem Blick. Kulturtheoretische Bezugnahmen werden größtenteils abstrakt diskutiert, aber in Ausführungen zum Unterricht, zu Lehrkräften sowie zu Schülerinnen und Schülern kaum weiter thematisiert. Die Herausforderung besteht darin, fachdidaktische Lehrbücher so zu gestalten, dass sie den Theorie-PraxisTransfer gezielt vor dem Hintergrund eines bedeutungsorientierten Kulturverständnisses theoretisch fundieren und methodisch anleiten. Dazu sollten die Metareflexionsaspekte Begründungspflicht und Kontextgebundenheit (Cramer et al. 2019) in kulturbezogener Hinsicht deutlicher als bisher in den Blick kommen: Es gilt angehende Lehrkräfte auf die begründende Offenlegung relevanter Auswahlkriterien von Ansätzen und Quellen in ihrer perspektivischen Unterrichtspraxis vorzubereiten (Cramer et al. 2019). Das setzt die Reflexion der eigenen kulturellen Kontextgebundenheit - wie auch der Schülerinnen- und Schülerkontexte - voraus, um individuelle Bedeutungszusammenhänge nicht auf- oder abzuwerten. In diesem Kontext ist die Integration biografietheoretischer und -didaktischer Zugänge mit Blick auf Kultur(en) von Bedeutung. Sie könnte einen Beitrag dazu leisten, dass Lehrende informelle Bildungszusammenhänge stärker erkennen, in die kulturreflexive Unterrichtspraxis einfließen lassen und reflexiv bearbeiten. 


\section{Anregungen für Forschung und Praxis}

Das entwickelte kulturbezogene Kategoriensystem hat sich für die Untersuchung kulturbezogener Diskursformationen in verschiedenen Fachdidaktiken als tragfähig erwiesen und kann eine Basis für weitere Forschungen zu Diskursen kultureller Bildung darstellen. Perspektivisch lassen sich mit diesem Kategoriensystem nicht nur kulturbezogene Diskurse von Fachdidaktiken untersuchen, sondern auch Dokumente kultureller Bildungsakteure (Kühn et al. 2020). Über die Englisch-, Geschichts- und Musikdidaktik hinausgehend, bietet es sich an, Lehrbücher weiterer Fachdidaktiken (z.B. Deutsch, Sport, Religion, Geografie etc.) oder der Erziehungswissenschaften hinsichtlich kulturtheoretischer Diskursformationen zu untersuchen.

Bisher geraten nur Schulbücher als kulturell tradierende Lehrmedien in den Blick. Vor diesem Hintergrund wird die Etablierung einer auf universitärer Ebene angesiedelten Lehrbuchforschung angeregt. Eine stärkere Wahrnehmung und In-BeziehungSetzung zur internationalen Schulbuchforschung scheint vielversprechend.

Die empirischen Befunde liefern Anregungen für die Weiterentwicklung von fachdidaktischen Lehrbüchern im Hinblick auf die konsequente Integration kultureller Hybridität oder kulturbezogener Selbstreflexion. Insbesondere für Passagen, die sich auf die unterrichtliche Praxis beziehen, ergeben sich vor dem Hintergrund der empirischen Ergebnisse zahlreiche Anregungen.

\section{Datenkorpus}

- A - Baumgärtner, U. (2015). Wegweiser Geschichtsdidaktik. Historisches Lernen in der Schule. Paderborn: Schöningh.

- B - Brauch, N. (2015). Geschichtsdidaktik. Oldenburg: De Gruyter.

- C-Günther-Arndt, H., \& Zülsdorf-Kersting, M. (Hrsg.) (2014/2003). GeschichtsDidaktik. Praxishandbuch für die Sekundarstufe I und II (6. akt. Aufl.). Berlin: Cornelsen.

- D - Pandel, H.-J. (2017/2013). Geschichtsdidaktik. Eine Theorie für die Praxis (2. Aufl.). Schwalbach a. T.: Wochenschau.

- E - Rohlfes, J. (2005/1986). Geschichte und ihre Didaktik (3. akt. Aufl.). Göttingen: Vandenhoeck \& Ruprecht.

- F - Böttger, H. (2005). Einführung in die Didaktik der englischen Sprache (Basiswissen Englischstudium Bd. 1). Tönning: Der andere Verlag.

- G - Gehring, W. (2010/1999). Englische Fachdidaktik. Theorien, Praxis, Forschendes Lernen (3. akt. Aufl., Grundlagen der Anglistik und Amerikanistik Bd. 20). Göttingen: Erich Schmidt.

- H - Haß, F. (2016/2006). Fachdidaktik Englisch: Tradition. Innovation. Praxis (2. akt. Aufl.). Stuttgart: Ernst Klett.

- I - Thaler, E. (2012). Englisch unterrichten. Grundlagen. Kompetenzen. Methoden. Berlin: Cornelsen.

- J - Timm, J.-P. (Hrsg.) (1998). Englisch lernen und lehren: Didaktik des Englischunterrichts. Berlin: Cornelsen. 
- K - Klippel, F., \& Doff, S. (2007). Englischdidaktik. Praxishandbuch für die Sekundärstufe I und II (4. Aufl.). Berlin: Cornelsen.

- L - Volkmann, L. (2010). Fachdidaktik Englisch: Kultur und Sprache. Tübingen: Narr.

- M - Dartsch, M., Knigge, J., Niessen, A., Platz, F., \& Stöger, C. (Hrsg.) (2018). Handbuch Musikpädagogik: Grundlagen - Forschung - Diskurse. Münster: Waxmann.

- N - Jank, W. (Hrsg.) (2005). Musikdidaktik. Praxishandbuch (7. Aufl.). Berlin: Cornelsen Scriptor.

- O - Kraemer, R.-D. (2007/2004). Musikpädagogik - Eine Einführung in das Studium (2. akt. Auflage). Forum Musikpädagogik (Bd. 55). Augsburg: Wißner.

- P - Schatt, P. W. (2007). Einführung in die Musikpädagogik. Einführung Erziehungswissenschaft. WBG.

Danksagung Wir danken dem Bundesministerium für Bildung und Forschung, das das Projekt „,Kultur in der Lehrerbildung (KulturLebi)“ unter der Fördernummer 01JK1602 in den Jahren 2017-2020 finanziell förderte.

Funding Open Access funding enabled and organized by Projekt DEAL.

Open Access Dieser Artikel wird unter der Creative Commons Namensnennung 4.0 International Lizenz veröffentlicht, welche die Nutzung, Vervielfältigung, Bearbeitung, Verbreitung und Wiedergabe in jeglichem Medium und Format erlaubt, sofern Sie den/die ursprünglichen Autor(en) und die Quelle ordnungsgemäß nennen, einen Link zur Creative Commons Lizenz beifügen und angeben, ob Änderungen vorgenommen wurden.

Die in diesem Artikel enthaltenen Bilder und sonstiges Drittmaterial unterliegen ebenfalls der genannten Creative Commons Lizenz, sofern sich aus der Abbildungslegende nichts anderes ergibt. Sofern das betreffende Material nicht unter der genannten Creative Commons Lizenz steht und die betreffende Handlung nicht nach gesetzlichen Vorschriften erlaubt ist, ist für die oben aufgeführten Weiterverwendungen des Materials die Einwilligung des jeweiligen Rechteinhabers einzuholen.

Weitere Details zur Lizenz entnehmen Sie bitte der Lizenzinformation auf http://creativecommons.org/ licenses/by/4.0/deed.de.

\section{Literatur}

Althans, B., \& Audehm, K. (2019). Bildung, Kultur, ästhetische Erfahrung? Eine Einladung zur begrifflichen Reflexion. Zeitschrift für Kulturwissenschaften, 13(2), 9-16.

Angermueller, J., Nonhoff, M., Herschinger, E., Macgilchrist, F., Reisigl, M., Wedl, J., Wrana, D., \& Ziem, A. (Hrsg.). (2014). Theorien, Methodologien und Kontroversen (Diskursforschung. Ein interdisziplinäres Handbuch, Bd. 1). Bielefeld: transcript.

Bauer, B., Beuter, K., Lindner, K., Hlukhovych, A., \& Vogt, S. (2018). Kulturbezogene Lehrerinnen- und Lehrerbildung. Grundlegungen, Perspektiven und Operationalisierungsoptionen. In B. Bauer, K. Beuter, K. Lindner, A. Hlukhovych \& S. Vogt (Hrsg.), Kultur und kulturelle Bildung (S. 13-36). Bamberg: UBP.

Beuter, K. (2019). Sprachen vermitteln, Welten sichten. Konzeptionalisierungen und Zusammenhänge von Sprache und Kultur in Bildungskontexten. In K. Beuter, A. Hlukhovych, K. Lindner \& S. Vogt (Hrsg.), Sprache und kulturelle Bildung (S. 15-33). Bamberg: UBP.

Bhabha, H. K. (2000). Die Verortung der Kultur. Tübingen: Stauffenburg.

Böhm, A. (2012). Theoretisches Grundieren: Textanalyse in der Grounded Theory. In U. Flick, E. v. Kardorff \& I. Steinke (Hrsg.), Qualitative Forschung. Ein Handbuch (Schriften zu Politik \& Kultur Bd. 4, 9. Aufl., S. 475-485). Reinbek bei Hamburg: rororo. 
Bourdieu, P. (2006). Wie die Kultur zum Bauern kommt. Über Bildung, Schule und Politik. Berlin: vsa.

Christophe, B. (2014). Kulturwissenschaftliche Schulbuchforschung - Trends, Ergebnisse und Potentiale (Eckert. Working Papers 2014/6). https://repository.gei.de/handle/11428/140. Zugegriffen: 20. Okt. 2020.

Costa, J., \& Drechsel, B. (2020). Künstlerisch-ästhetische Praxen angehender Lehrkräfte. Befunde einer quantitativen Sekundäranalyse. In S. Timm, J. Costa, C. Kühn \& A. Scheunpflug (Hrsg.), Kulturelle Bildung. Theoretische Perspektiven, methodologische Herausforderungen, empirische Befunde (S. 129-146). Münster: Waxmann.

Costa, J., Kühn, C., Timm, S., \& Franken, L. (2018). Kulturelle Lehrerbildung - Professionalität im Horizont der Globalisierung. Zeitschrift für internationale Bildungsforschung und Entwicklungspädagogik (ZEP), 41(4/4), 20-29.

Cramer, C., Harant, M., Merk, S., Drahmann, M., \& Emmerich, M. (2019). Meta-Reflexivität und Professionalität im Lehrerinnen- und Lehrerberuf. Zeitschrift für Pädagogik, 65(3), 401-423.

Dumm, S., \& Niekler, A. (2016). Methoden, Qualitätssicherung und Forschungsdesign. In M. Lemke \& G. Weidemann (Hrsg.), Text Mining in den Sozialwissenschaften. Grundlagen und Anwendungen zwischen qualitativer und quantitativer Diskursanalysen (S. 89-116). Wiesbaden: VS.

Fäcke, C. (2016). Lehrwerkforschung - Lehrwerkgestaltung - Lehrwerkrezeption, Überlegungen zur Relevanz von Lehrwerken für den Fremdsprachenunterricht. In M. Rückl (Hrsg.), Sprachen und Kulturen: vermitteln und vernetzen. Beiträge zu Mehrsprachigkeit und Inter-/Transkulturalität im Unterricht, in Lehrwerken und in der Lehrer/innen/bildung (S. 34-48). Münster: Waxmann.

Fend, H. (1980). Theorie der Schule. Weinheim: Beltz.

Flick, U., v. Kardoff, E., \& Steinke, I. (Hrsg.). (2013). Qualitative Forschung. Ein Handbuch (10. Aufl.). Reinbeck bei Hamburg: Rowohlt.

Foucault, M. (1991). Die Ordnung des Diskurses. Frankfurt a. M.: S. Fischer.

Franken, L., \& Lindner, K. (2019). Kulturbezogene Bildung und kulturelles Erbe in der Lehrerbildung der Geschichtsdidaktik. In K. C. Schüppel \& B. Welzel (Hrsg.), Sharing Heritage. Objekte, Prozesse, Akteure (S. 185-197). Berlin: Reimer.

Fuchs, M. (2008). Kultur Macht Sinn. Einführung in die Kulturtheorie. Wiesbaden: Springer.

Fuchs, S. (2011). Aktuelle Entwicklungen in der der schulbuchbezogenen Forschungen in Europa. Zeitschrift für Bildung und Erziehung, 64(1/4), 5-20.

Fuchs, M. (2019). Einführung in Konzept und Grundlagen von kultureller Schulentwicklung. https://www. kubi-online.de/. Zugegriffen: 20. Okt. 2020.

Geertz, C. (2010). Dichte Beschreibung. Beiträge zum Verstehen kultureller Systeme. Frankfurt a. M.: Suhrkamp.

Glaser, B. G., \& Strauss, A. L. (2010). Grounded Theory. Strategien qualitativer Forschung. Bern: Huber.

Göhlich, M. (2010). Homi K. Bhabha: Die Verortung der Kultur. Kontexte und Spuren einer postkolonialen Identitätstheorie. In B. Jörissen \& J. Zirfas (Hrsg.), Schlüsselwerke der Identitätsforschung (S. 315-330). Wiesbaden: VS.

Grawan, F. (2014). Impliziter Rassismus und kulturelle Hegemonie im Schulbuch? Rassismuskritische Analyse und objektiv hermeneutische Rekonstruktion (Eckert Working Papers 2014/2). https:// repository.gei.de/handle/11428/137. Zugegriffen: 20. Okt. 2020.

Griese, H. (2006). „Meine Kultur mache ich selbst.“ Kritik der Inter- und Transkulturalität in Zeiten der Individualisierung und Globalisierung. Zeitschrift für internationale Bildungsforschung und Entwicklungspädagogik (ZEP), 29(4/4), 19-23.

Herder, J.G. (2010). Abhandlung über den Ursprung der Sprache. In R. Borgards (Hrsg.), Texte zur Kulturtheorie und Kulturwissenschaft. Stuttgart: Reclam.

Jahn, E. (2008). Denglisch statt deutsch? Zur Veränderung des Sprachverhaltens in Deutschland. In E. Jahn (Hrsg.), Politische Streitfragen (S. 115-131). Wiesbaden: VS.

Kaiser, H.J. (2018). Legitimationen musikpädagogischen Handelns in Deutschland. In M. Dartsch, J. Knigge, A. Niessen, F. Platz \& C. Stöger (Hrsg.), Handbuch Musikpädagogik: Grundlagen Forschung - Diskurse (S. 38-43). Münster: Waxmann.

Keller, R. (2011a). Diskursforschung. Eine Einführung für SozialwissenschaftlerInnen. Wiesbaden: VS.

Keller, R. (2011b). Wissenssoziologische Diskursanalyse. Grundlegung eines Forschungsprogramms. Wiesbaden: VS.

Klepacki, L. (2012). Warum eigentlich Kulturelle Bildung? Reflexive Ansätze zu einer disziplinären Selbstvergewisserung aus geisteswissenschaftlicher Perspektive. In T. Fink, B. Hill, V.-I. Reinwand \& A. Wenzlik (Hrsg.), Die Kunst, über Kulturelle Bildung zu forschen. Theorie- und Forschungsansätze (S. 23-35). München: kopaed. 
Klepacki, L. (2014). Lehrerbildung als Kulturelle Bildung - ein kulturtheoretisch-geisteswissenschaftliches Essay über Schule. https://www.kubi-online.de/. Zugegriffen: 20. Okt. 2020.

Klopsch, B. (2019). Schulkultur durch kulturelle Schulentwicklung gestalten: Von der Möglichkeit, lernförderliche Haltungen zu entwickeln. https://www.kubi-online.de/. Zugegriffen: 20. Okt. 2020.

Kühn, C., Lindner, K., \& Scheunpflug, A. (2020). Kultur und kulturelle Bildung in der universitären Lehrerbildung. Lehrbücher als Quellen diskursprägender Aspekte. In S. Timm, J. Costa, C. Kühn \& A. Scheunpflug (Hrsg.), Kulturelle Bildung. Theoretische Perspektiven, methodologische Herausforderungen, empirische Befunde (S. 213-228). Münster: Waxmann.

Lange, H., \& Sinning, S. (Hrsg.). (2014a). Ästhetik und Leiblichkeit. Fachdidaktik und Themenkonstitution in ästhetisch-leiblichen Fächern und Lernbereichen. Hohengehren: Schneider.

Lange, H., \& Sinning, S. (Hrsg.). (2014b). Kultur und Gesellschaft. Fachdidaktik und Themenkonstitution in den geistes- und sozialwissenschaftlichen Fächern und Lernbereichen. Hohengehren: Schneider.

Leifert, B. (2012). Lesarten des Geschlechts und Geschlechterverhältnisse - Eine Analyse des sozialwissenschaftlichen Arbeitsbuches ,Politik/Wirtschaft 5/6` von Franz Josef Floren mithilfe der Objektiven Hermeneutik. Politisches, 12(1/2), 40-47.

Liebau, E., Jörissen, B., Hartmann, S., Lohwasser, D., Werner, F., Klepacki, L., \& Wagner, E. (2013). Forschungen zur Kulturellen Bildung Bestand und Perspektiven. Projektbericht. Berlin: BMBF.

Liebau, E., Jörissen, B., \& Klepacki, L. (2014). Teil 3: Was wir sehen und was wir nicht sehen. In E. Liebau, B. Jörissen \& L. Klepacki (Hrsg.), Forschung zur kulturellen Bildung. Grundlagenreflexionen und empirische Befunde (S. 177-216). München: kopaed.

Luckmann, T. (2006). Die kommunikative Konstruktion der Wirklichkeit. In D. Tänzler, H. Knoblauch \& H.-G. Soeffner (Hrsg.), Neue Perspektiven der Wissenssoziologie (S. 15-26). Konstanz: UVK.

Mayring, P. (2015). Qualitative Inhaltsanalyse Grundlagen und Techniken (11. Aufl.). Weinheim: Beltz.

Reckwitz, A. (2000). Die Transformation der Kulturtheorien. Zur Entwicklung eines Theorieprogramms. Weilerswist: Velbrück.

Reckwitz, A. (2008). Unscharfe Grenzen. Perspektiven der Kultursoziologie. Bielefeld: transcript.

Reckwitz, A. (2011). Die Kontingenzperspektive der „Kultur“. Kulturbegriffe, Kulturtheorien und das kulturwissenschaftliche Forschungsprogramm. In F. Jaeger \& B. Liebsch (Hrsg.), Handbuch Kulturwissenschaften (S. 1-20). Stuttgart: Metzler.

Reckwitz, A. (2017). Die Gesellschaft der Singularitäten. Zum Strukturwandel der Moderne. Frankfurt a. M.: Suhrkamp.

Sattler, S. (2014). Computergestützte qualitative Datenerhebung. In C. Bischoff, K. Oehme-Jüngling \& W. Leimgruber (Hrsg.), Methoden der Kulturanthropologie (S. 467-487). Bern: UTB.

Scheunpflug, A., Franz, J., \& Stadler-Altmann, U. (2012). Zur „Kultur“ in pädagogischen Zusammenhängen. In T. Fink, B. Hill, V. I. Reinwand \& A. Wenzlik (Hrsg.), Die Kunst, über Kulturelle Bildung zu forschen. Theorie- und Forschungsansätze (S. 99-109). München: kopaed.

Spivak, G.C. (1988). Can the subaltern speak? In C. Nelson \& L. Grossberg (Hrsg.), Marxism and the interpretation of culture (S. 271-313). Urbana: University of Illinois Press.

Stute, D., \& Wibbing, G. (2014). Kulturelle Bildung als Baustein der Unterrichtsentwicklung. https://www. kubi-online.de/. Zugegriffen: 20. Okt. 2020.

Timm, S., \& Scheunpflug, A. (2020). Orientierungen im Feld kulturellen Professionshandelns. Empirische Einblicke und Konsequenzen für die Lehrkräftebildung. In S. Timm, J. Costa, C. Kühn \& A. Scheunpflug (Hrsg.), Kulturelle Bildung. Theoretische Perspektiven, methodologische Herausforderungen, empirische Befunde (S. 147-161). Münster: Waxmann.

UNESCO \& GEI (2018). Vereinte Nationen für Bildung, Wissenschaft und Kultur \& Georg-Eckert Institut Leibniz Institut für internationale Schulforschung (Hrsg.) (2018). Schulbuchinhalte inklusiv gestalten: Religion, Gender und Kultur im Fokus. https://www.unesco.de/sites/default/files/2018-09/ Schulbuchinhalte\%20inklusiv\%20gestalten.pdf. Zugegriffen: 20. Okt. 2020.

Viebrock, B. (2008). Englischdidaktik im Spiegel neuer Einführungswerke. Ein thematischer Vergleich. Zeitschrift für Fremdsprachenforschung, 19(1/2), 83-120.

Welsch, W. (2010). Was ist eigentlich Transkulturalität? In L. Darowska, T. Lüttenberg \& C. Machold (Hrsg.), Hochschule als transkultureller Raum (S. 39-66). Bielefeld: transcript.

Wulf, C. (2004). Anthropologie. Geschichte, Kultur, Philosophie. Reinbek: Rowohlt. 